ВІСНИК

ОДЕСЬКОГО НАЦІОНАЛЬНОГО

МОРСЬКОГО УНІВЕРСИТЕТУ
HERALD

OF THE ODESSA NATIONAL

MARITIME UNIVERSITY № 1 (64), 2021

УДК 620:178.3

DOI 10.47049/2226-1893-2021-1-149-156

\title{
ОПРЕДЕЛЕНИЕ ПРЕДЕЛА ВЫНОСЛИВОСТИ ДЕТАЛЕЙ ПО ПАРАМЕТРАМ НАКЛОННОГО УЧАСТКА КРИВОЙ УСТАЛОСТИ
}

\author{
В.В. Галевский \\ ст. преподаватель кафедры «Машиноведение»
}

Одесский национальный морской университет, Одесса, Украина

Аннотация. В статье рассмотрена задача, связанная с ускоренным определением предела выносливости стальных объектов.

Предложено для её решения использовать наклонный участок кривой усталости в логарифмических координатах, построенный по классической методике согласно степенному уравнению. При этом предел выносливости предлагается находить по параметрам уравнения кривой усталости, выраженной уравнением Вейбулла, получаемым пересчётом.

Экспериментальная проверка предложенного подхода на гладких и надрезанных образцах, изготовленных из стали 45, показала, что погрешности предложенного метода находятся в допустимых пределах.

Ключевые слова: ускоренные испытания, предел выносливости, параметры наклонного участка.

УДК 620:178.3

DOI 10.47049/2226-1893-2021-1-149-156

\section{ВИЗНАЧЕННЯ МЕЖІ ВИТРИВАЛОСТІ ДЕТАЛЕЙ ЗА ПАРАМЕТРАМИ ПОХИЛОЇ ДІЛЯНКИ КРИВОЇ ВТОМИ}

\section{В.В. Галевський}

ст. викладач кафедри «Машинознавство»

Одеський національний морський університет, Украӥна, Одеса

Анотація. У статті розглянута задача, пов'язана з прискореним визначенням межі витривалості сталевих об'єктів.

Запропоновано для їі вирішення використовувати похилу ділянку кривої втоми в логарифмічних координатах, побудовану за класичною методикою згідно до степеневого рівняння. При иьвому межу витривалості пропонується знаходити за параметрами рівняння кривої втоми, вираженої рівнянням Вейбула, що одержується перерахунком.

Експериментальна перевірка запропонованого підходу на гладких $i$ надрізаних зразках, виготовлених зі сталі 45, показала, що похибки запропонованого методу знаходяться в допустимих межах.

Ключові слова: прискорені випробування, межа витривалості, параметри похилої ділянки.

() Галевский В.В., 2021 
UDC 620:178.3

DOI 10.47049/2226-1893-2021-1-149-156

\title{
DETERMINATION OF THE ENDURANCE LIMIT OF PARTS BY THE PARAMETERS OF THE INCLINED SECTION OF THE FATIGUE CURVE
}

\author{
V.V. Halevskyi \\ Senior Lecturer of the Department «Machine Science» \\ Odessa National Maritime University, Ukraine, Odessa
}

\begin{abstract}
The article deals with the problem associated with the acelerated determination of the endurance limit of steel objects.

It is proposed to use the sloped section of the fatigue curve in logarithmic coordinates to solve this problem, constructed according to the classical technique according to the power equation. In this case, the endurance limit is proposed to be found by the parameters of the fatigue curve equation, expressed by the Weibull equation, obtained by recalculation.

Experimental verification of the proposed approach on smooth and notched specimens made of steel 45 showed that the errors of the proposed method are within acceptable limits.
\end{abstract}

Keywords: accelerated tests, endurance limit, parameters of an inclined section.

Введение. Целью большинства методов ускоренных испытаний на сопротивление усталости является оценка предела выносливости. Среди них существует ряд методов, основанных на использовании корреляционных связей межу параметрами наклонного участка кривой усталости и пределом выносливости [1]. При этом база, на которой он определяется, составляет, как правило, $10^{7}$ циклов. Исключением являются методы, в основе которых лежит уравнение Вейбулла. Между тем известно, что пределы выносливости некоторых объектов (например, деталей крупных размеров, соединений с гарантированным натягом и др.) определяются на базах, превышающих указанную. Данный факт привёл к появлению методов, основанных на использовании составных кривых усталости [2]. В отличие от них рассматриваемый в статье подход не предполагает построение составной кривой усталости, а основан на пересчёте параметров степенного уравнения в параметры уравнения Вейбулла.

Целью статьи является разработка ускоренного метода определения предела выносливости, основанного на пересчёте параметров степенного уравнения в параметры уравнения Вейбулла.

Изложение основного материала. Степенное уравнение кривой усталости и уравнение Вейбулла представим в виде 


$$
\begin{gathered}
\sigma^{m_{C}} N=10^{C_{C}}, \\
\left(\sigma-\sigma_{R}\right)^{m_{W}} N=10^{C_{W}},
\end{gathered}
$$

где $\sigma$ и $N$ - текущие напряжение и число циклов до разрушения соответственно; $\sigma_{R}$ - предел выносливости; $m_{C}, C_{C}, m_{W}$ и $C_{W}$ - параметры.

Для пересчёта параметров $m_{C}, C_{C}$, в параметры $m_{W}$ и $C_{W}$, воспользуемся их корреляционными зависимостями с пределом выносливости [1]

$$
\begin{gathered}
m_{C}=f_{C}\left(\sigma_{R}\right)=0,027 \sigma_{R}+1,4 ; \\
C_{C}=F_{C}\left(\sigma_{R}\right)=0,997\left(m_{C}+1\right) \lg \sigma_{R}+4,0 ; \\
m_{W}=f_{W}\left(\sigma_{R}\right)=\frac{1}{5,254 \sigma_{R}^{0,01}-5,038}-1,0 ; \\
C_{W}=F_{W}\left(\sigma_{R}\right)=\lg \frac{\left(7,029 \sigma_{R}+3599\right)^{m_{W}+1}}{m_{W}+1} .
\end{gathered}
$$

Найденные по зависимостям (3)-(6) значения параметров $m_{C}, C_{C}$, $m_{W}$ и $C_{W}$ для пределов выносливости $\sigma_{R}=100-, 500$ МПа представлены в табл. 1.

Таблий 1

Расчётные значения параметров $m_{C}, C_{C}, m_{W} u C_{W}$

\begin{tabular}{|c|c|c|c|c|c|}
\hline$\sigma_{R}$, МПа & 100 & 200 & 300 & 400 & 500 \\
\hline$m_{C}$ & 4,1 & 6,8 & 9,5 & 12,2 & 14,9 \\
\hline$C_{C}$ & 14,17 & 21,89 & 29,93 & 38,24 & 46,78 \\
\hline$m_{W}$ & 1,16 & 0,99 & 0,91 & 0,85 & 0,81 \\
\hline$C_{W}$ & 7,50 & 7,07 & 6,88 & 6,78 & 6,71 \\
\hline
\end{tabular}

Используя данные табл. 1, построим графики зависимости $m_{W}\left(m_{C}\right)$ и $C_{W}\left(C_{C}\right)$ (рис. 1,2$)$.

Уравнения аппроксимирующих прямых, полученных по методу наименьших квадратов, имеют вид

$$
\begin{gathered}
m_{W}=1,238-0,031 m_{C} . \\
C_{W}=7,681-0,022 C_{C} .
\end{gathered}
$$




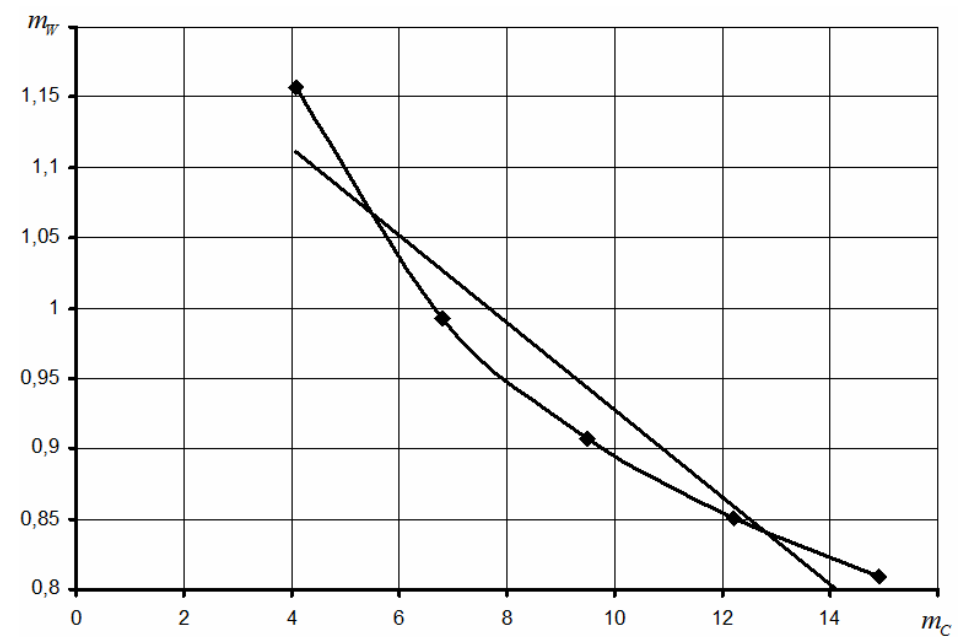

Рис. 1. Зависимость $m_{W}\left(m_{C}\right)$

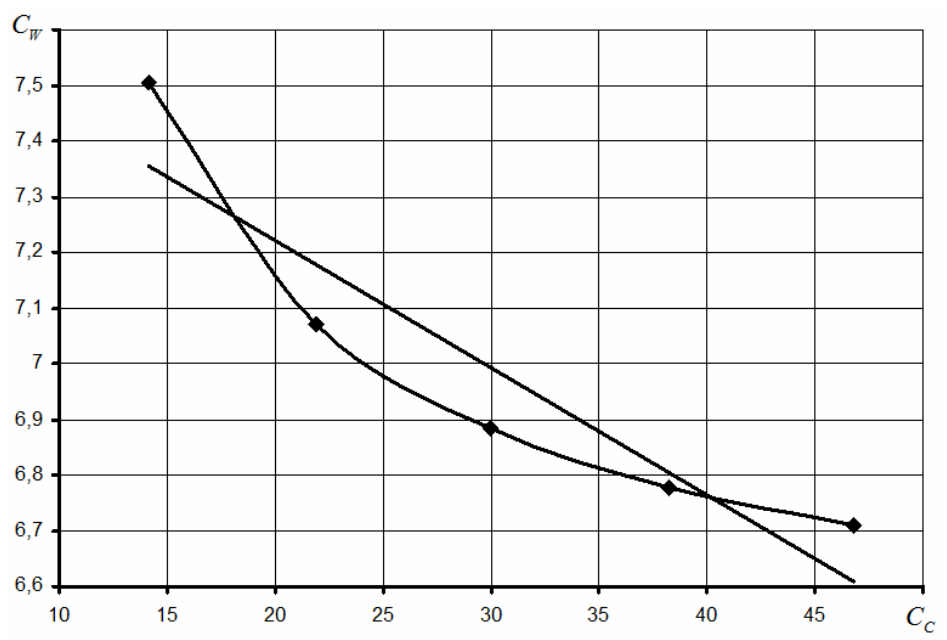

Рис. 2. Зависимость $C_{W}\left(C_{C}\right)$

Коэффициенты корреляции $R^{2}$ величин $m_{W}$ и $m_{C}$, а также $C_{W}$ и $C_{C}$ в уравнениях (7) и (8) соответственно составили 0,92 и 0,86 , что свидетельствует о тесной связи между этими параметрами.

Для экспериментальной проверки полученных зависимостей обратимся к результатам испытаний лабораторных образцов из стали 45 (гладких и надрезанных) при регулярной нагрузке, которые приведены в работе [3] (табл. 2). Форма образцов и их геометрические параметры представлены на рис. 3. и в табл. 2. 


\begin{tabular}{c|c} 
ВІСНИК & HERALD \\
$\begin{array}{c}\text { ОДЕСЬКОГО НАЦІОНАЛЬНОГО } \\
\text { МОРСЬКОГО УНІВЕРСИТЕТУ } \\
\text { № } 1 \text { (64), 2021 }\end{array}$ & OF THE ODESSA NATIONAL \\
MARITIME UNIVERSITY \\
№ 1 (64), 2021
\end{tabular}

a)

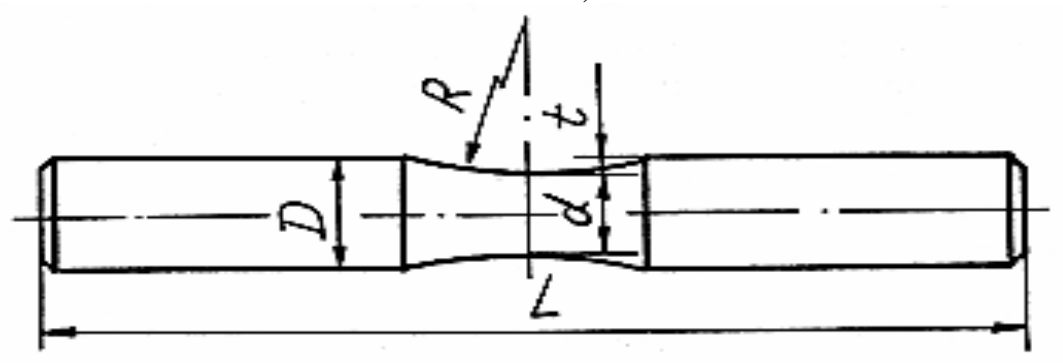

б)

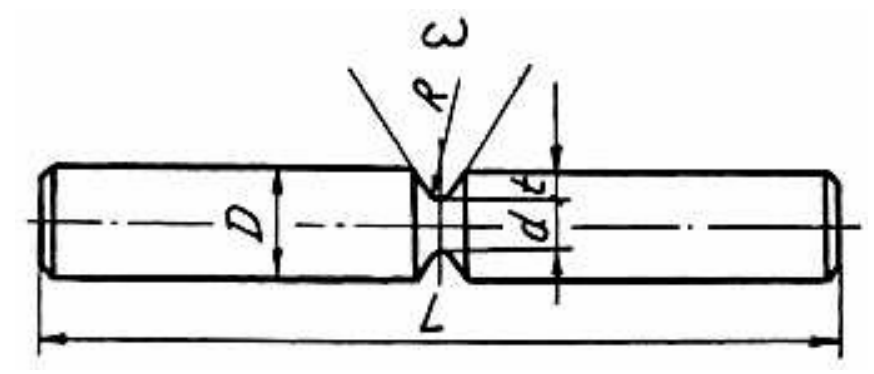

Рис. 3. Конструкции образиов:

$a$ - гладкий образеи; б - образец с надрезом

Таблийа 2

Размеры гладких и надрезанных образиов

\begin{tabular}{|c|c|c|c|c|c|c|c|}
\hline \multirow{2}{*}{$\begin{array}{c}\text { Тип } \\
\text { образца }\end{array}$} & \multirow{2}{*}{$\begin{array}{c}\text { Форма } \\
\text { рабочей } \\
\text { части }\end{array}$} & \multicolumn{5}{|c|}{ Размеры, мм } & \multirow[b]{2}{*}{$\omega$} \\
\hline & & $\mathrm{L}$ & $D$ & $d$ & $t$ & $R$ & \\
\hline $\mathrm{I}$ & гладкий & 226 & 12 & 10 & 1,0 & 50,0 & - \\
\hline II & с надрезом & 226 & 12 & 10 & 1,0 & 2,0 & - \\
\hline
\end{tabular}

В результате обработки данных таблицы 3 по методу наименьших квадратов параметры $m_{C}$ и $C_{C}$ для гладких образцов составили 18,18 и 50,04, а для надрезанных 15,97 и 37,01 соответственно. Пределы выносливости, найденные с помощью метода «вверх - вниз», оказались равными 250 МПа (для гладких образцов), и 204,0 МПа (для надрезанных образцов). 
Таблица 3

Результаты испытаний гладких лабораторных образцов из стали 45 при стационарной нагрузке [3]

\begin{tabular}{|c|c|c|c|c|}
\hline Номер п/п & $\sigma$, МПа & $\lg \sigma$ & $N$, цикл & $\lg N$ \\
\hline 1 & \multirow{5}{*}{300} & \multirow{5}{*}{2,477} & 72200 & 4,859 \\
\hline 2 & & & 86200 & 4,936 \\
\hline 3 & & & 98300 & 4,993 \\
\hline 4 & & & 113300 & 5,054 \\
\hline 5 & & & 199200 & 5,299 \\
\hline 6 & \multirow{5}{*}{285} & \multirow{5}{*}{2,455} & 205800 & 5,316 \\
\hline 7 & & & 206800 & 5,316 \\
\hline 8 & & & 299100 & 5,476 \\
\hline 9 & & & 308900 & 5,490 \\
\hline 10 & & & 394400 & 5,596 \\
\hline 11 & \multirow{5}{*}{270} & \multirow{5}{*}{2,431} & 336600 & 5,527 \\
\hline 12 & & & 362400 & 5,559 \\
\hline 13 & & & 547100 & 5,738 \\
\hline 14 & & & 602600 & 5,780 \\
\hline 15 & & & 703600 & 5,847 \\
\hline
\end{tabular}

Параметры $m_{W}$ и $C_{W}$, найденные по формулам (7) и (8) составили для гладких образцов 0,73 и 6,68 , а для надрезанных 0,83 и 6,87 .

Результаты ускоренных испытаний этих же образцов при скоростях увеличения нагрузки $\alpha=100$ Па/цикл и $\alpha=200$ Па/цикл приведены в табл. 4 и 5.

По данным таблиц 4 и 5 определены значения пределов выносливости $\sigma_{R i}^{y C}$ и $\bar{\sigma}_{R}^{y C}$ по формулам

$$
\begin{gathered}
\sigma_{R i}^{y C}=\sigma_{P}-\left[\alpha\left(m_{W}+1\right) \cdot 10^{C_{W}}\right]^{\frac{1}{m_{W}+1}} ; \\
\bar{\sigma}_{R}^{y C}=\frac{1}{n} \sum_{i=1}^{n} \sigma_{R i}^{y C} .
\end{gathered}
$$

В результате расчёта погрешности ускоренного определения предела выносливости для гадких образцов составили - 0,78 \% (при $\alpha=100$ Па/цикл) и 6,33 \% (при $\alpha=200$ Па/цикл). Для надрезанных $0,61 \%$ (при $\alpha=100$ Па/цикл) и 4,27 \% (при $\alpha=200$ Па/цикл). Эти погрешности характеризуют общую ошибку метода и включают как систематическую, так и случайную составляющую. Их можно считать приемлемыми, поскольку они не превышают допустимых значений. 
Результаты ускоренных испьтаний

Таблища 4 гладких образиов и расчёта значений $\sigma_{R}^{y C}$ и $\bar{\sigma}_{R}^{y C}$

\begin{tabular}{|c|c|c|c|c|c|}
\hline $\begin{array}{l}\text { Номер } \\
\Pi / \Pi\end{array}$ & $\alpha$, Па/цикл & $\sigma_{1}$, МПа & $\sigma_{P}, \mathrm{MПа}$ & $\begin{array}{l}\sigma_{R i}^{y C}, \\
\text { МПа }\end{array}$ & $\begin{array}{l}\bar{\sigma}_{R}^{y C}, \\
\text { МПа }\end{array}$ \\
\hline 1 & \multirow{5}{*}{100} & \multirow{5}{*}{240} & 293,5 & 244,9 & \multirow{5}{*}{250,6} \\
\hline 2 & & & 294,1 & 245,5 & \\
\hline 3 & & & 299,4 & 250,8 & \\
\hline 4 & & & 300,8 & 252,2 & \\
\hline 5 & & & 308,3 & $\frac{259,7}{259,7}$ & \\
\hline 6 & \multirow{5}{*}{200} & \multirow{5}{*}{225} & 296,9 & 224,4 & \multirow{5}{*}{233,2} \\
\hline 7 & & & 307,3 & 234,8 & \\
\hline 8 & & & 307,5 & 235,0 & \\
\hline 9 & & & 308,2 & 235,7 & \\
\hline 10 & & & 308,6 & 236,1 & \\
\hline
\end{tabular}

Таблий 5

Результаты ускоренных испьттаний образиов

с концентратором напряжений и расчёта значений $\sigma_{R}^{y C} u \bar{\sigma}_{R}^{y C}$

\begin{tabular}{|c|c|c|c|c|c|}
\hline $\begin{array}{c}\text { Номер } \\
\Pi / \Pi \\
\end{array}$ & $\begin{array}{c}\alpha, \\
\text { Па/цикл }\end{array}$ & $\sigma_{1}$, МПа & $\sigma_{P}$, МПа & $\begin{array}{l}\sigma_{R i}^{y C}, \\
\text { МПа }\end{array}$ & $\begin{array}{l}\bar{\sigma}_{R}^{V C}, \\
\text { МПа }\end{array}$ \\
\hline 1 & \multirow{5}{*}{100} & \multirow{5}{*}{180} & 250,3 & 199,0 & \multirow{5}{*}{214,6} \\
\hline 2 & & & 258,7 & 207,4 & \\
\hline 3 & & & 270,8 & 219,5 & \\
\hline 4 & & & 272,1 & 220,8 & \\
\hline 5 & & & 277,8 & 226,5 & \\
\hline 6 & \multirow{5}{*}{200} & \multirow{5}{*}{180} & 280,5 & 205,7 & \multirow{5}{*}{210,8} \\
\hline 7 & & & 285,5 & 210,7 & \\
\hline 8 & & & 286,2 & 211,4 & \\
\hline 9 & & & 288,0 & 213,2 & \\
\hline 10 & & & 288,0 & 213,2 & \\
\hline
\end{tabular}

\section{Выводы}

1. Предложен новый способ пересчёта параметров степенного уравнения кривой усталости в параметры уравнения Вейбулла.

2. На основе этого способа разработан метод ускоренного определения предела выносливости стальных объектов.

3. Экспериментальная проверка метода показала, что его погрешность находится в допустимых пределах. 


\section{ЛІТЕРАТУРА}

1. Олейник Н.В., Скляр С.П. Ускоренные испьтания на усталость. - К.: Наук. думка, 1985. - 304 c.

2. Олейник Н.В., Кибаков А.Г. Оперативная оценка сопротивления усталости материалов и деталей. - Одесса: Астропринт, 1998. $-142 c$.

3. Олейник Н.В., Коноплёв А.В., Кибаков А.Г. Методь ускоренного определения характеристик сопротивления усталости в практических приложениях. - Одесса: Астропринт, 2000. $138 \mathrm{c}$.

\section{REFERENCES}

1. Oleinik, N.V., Sklyar, S.P. Uskorennie ispitaniya na ustalost' [Accelerated Fatigue Tests]. - K.: Naukova dumka (Scientific thought), 1985. - 304 l. [in Russian].

2. Oleinik, N.V., Kibakov, A.G. Operativnaya ocenka soprotivleniya ustalosti materialov $i$ detalei [Rapid assessment of fatigue resistance of materials and parts]. - Odessa: Astroprint, 1998. $142 l$.

3. Oleinik, N.V., Konoplev, A.V., Kibakov, A.G. Metodi uskorennogo opredeleniya harakteristik soprotivleniya ustalosti $v$ prakticheskih prilogheniyah [Methods of accelerated determination of fatigue resistance characteristics in practical applications]. - Odessa: Astroprint, 2000. - 138 l.

Стаття надійшла до редакиії 26.03.2021

Посилання на статтю: Галевский В.В. Определение предела выносливости деталей по параметрам наклонного участка кривой усталости // Вісник Одеського національного морського університету: Зб. наук. праць, 2021. № 1 (64). С. 149-156. DOI 10.47049/ 2226-1893-2021-1-149-156.

Article received 26.03.2021

Reference a JournalArtic: Halevskyi V.V. Determination of the endurance limit of parts by the parameters of the inclined section of the fatigue curve // Herald of the Odessa National Maritime University. 2021. 1(64), 149-156. DOI 10.47049/22261893-2021-1-149-156. 